\title{
Meta fuzzy functions: Application of recurrent type-1 fuzzy functions
}

\author{
Nihat Tak* \\ Kirklareli University, Turkey \\ Ortabayir Mahallesi Caner Sokak No: 14/2 Kagithane, Istanbul, Turkey
}

\section{H I G H L I G H T S}

- MFFs are the first approach that aims to aggregate methods in functions by using FCM.

- The assumption of the MFFs is that a method has some information for a given dataset.

- The only need for applying the proposed method is to understand the FCM algorithm.

- MFFs gives more accurate results by aggregating the related methods in functions.

\section{A R T I C L E I N F O}

\section{Article history:}

Received 25 January 2018

Received in revised form 30 July 2018

Accepted 7 August 2018

Available online $\mathrm{xxxx}$

\section{MSC:}

$00-01$

99-00

\section{Keywords:}

Fuzzy C-means clustering

Meta-analysis

Type-1 fuzzy functions

Hybridizing

Forecasting

\begin{abstract}
A B S T R A C T
The main objective of meta-analysis is to aggregate the results of multiple scientific studies on a specific topic. Instead of aggregating the results of different studies, different methods are aggregated with the help of fuzzy c-means clustering algorithm in the proposed method. Meta fuzzy functions are introduced in the paper. The idea of meta fuzzy functions is to aggregate the methods which are proposed for the same purpose; forecasting, prediction, etc. The study aggregates the models for the same method under different parameter specifications rather than aggregating different methods. Recently, recurrent type-1 fuzzy functions are introduced as an alternative forecasting method. The main advantages of recurrent type- 1 fuzzy functions are that they are free of assumptions and rules. There are three parameters to be adjusted for recurrent type- 1 fuzzy functions; the number of lags for $A R(p)$, the number of lags for MA(q), and the number of clusters. The models for recurrent type- 1 fuzzy functions with different parameter specifications are aggregated in the paper. The results show that it is possible to increase the forecasting performances of recurrent type- 1 fuzzy functions in terms of both RMSE and MAPE.
\end{abstract}

(C) 2018 Elsevier B.V. All rights reserved.

\section{Introduction}

In this study, Meta Fuzzy Functions (MFFs) are introduced. One of the motivations of the study is the concept of meta-analysis. Meta-analysis was introduced by Glass [1] in 1976. He statistically aggregated the findings of 375 different psychotherapy outcome studies in his paper. Also, DerSimonian and Laird [2] defined that meta-analysis is a collection of analytic results for integrating the findings. The studies based on meta-analysis have become more popular in the last few decades. In the study, rather than aggregating the results of different studies for a purpose, the different methods for a purpose are aggregated. It has been shown that aggregation or hybridizing different methods have better forecasting or prediction accuracy. Thus, the main contribution of the paper is to aggregate as many methods as we can collect in functions. Aggregation of the methods is proceeded in the same sense of meta-analysis by looking at the outcomes/results of the methods.

\footnotetext{
* Correspondence to: Kirklareli University, Turkey.

E-mail address: nihattak@gmail.com.
}

Aggregation of the methods are performed by using Fuzzy C-Means (FCM) clustering technique in the study. This is a first study in the literature that aims to aggregate methods in the notion of metaanalysis by using FCM.

The proposed method is formed with a set of outcomes of the methods for a specific topic, such as forecasting performances of methods for a time series dataset. The outcomes of the methods are clustered using Fuzzy C-Means (FCM) clustering algorithm which was introduced by Bezdek [3]. Using the degrees of memberships values of the methods for each cluster, MFFs are obtained. Finally, the function that has the best set of outcomes is selected as the best MFF. As an application, a forecasting method, Recurrent Type1 Fuzzy Functions (R-T1FF), which was introduced by Tak et al. [4] is used.

T1FFs were introduced for classification and regression problems at first by Turksen [5] in 2008. First, Beyhan and Alici [6] and later, Aladag et al. [7] adapted T1FFs to time series forecasting problems. Beyhan and Alici [6] used an auto-regressive with exogenous input (ARX) model structure that was not able to search for the best model. Therefore, Aladag et al. [7] proposed a forecasting method 
to search for the best model by adapting Autoregressive (AR) model into their algorithm. Tak et al. [4] in 2017 introduced another method with T1FFs including also Moving Average (MA) model with recurrent learning structure into their algorithm. Eventually, R-T1FFs outperformed other two methods, which use T1FFs, in terms of RMSE values.

The purpose of the study is to aggregate the methods for the same purpose into functions. The idea behind aggregating the methods is the assumption that each method has much, partial or no information for a given dataset. Thus, while the methods that perform better will be collected into one function, the methods that perform worse will be collected into another function. Our aim is to obtain better outcomes using the power of many methods or, at least, obtain the best method among many.

The remainder of this article is organized as follows. In the second section, two algorithms are given; MFFs and MFFs of T1FFs. In order to evaluate the performance of the proposed method, some applications are given in the third section. Finally, the conclusions and remarks are given in Section 4.

\section{Proposed method}

The questions like "Which method should we choose for a dataset?" and "Please explain why the proposed method does not outperform other methods?" lead us to come up with the proposed method, MFFs. The main scope of the proposed method is to aggregate as many methods as we can collect into functions and, eventually, to get better outcomes. Thus, the input matrix of the MFFs consists of the outcomes of the methods which were previously introduced. Using the outcomes of the methods as their characteristics, the methods are clustered by using FCM. Finally, the best cluster (function) is selected as the $M F F_{\text {best }}$. Although there are different fuzzy clustering techniques such as FCM [3], Possibilistic C-Means (PCM) [8], Fuzzy Possibilistic C-Means (FPCM) [9], Possibilistic Fuzzy C-Means (PFCM) [9], and Interval Type-2 Fuzzy Possibilistic C-Means (IT2FPCM) [10], FCM is preferred because of its fame and simplicity. In other words, FCM is just one way to obtain the weights of the methods in functions. Using another fuzzy clustering technique in the setup of the MFFs might be the future work.

Two algorithms are introduced in this section. The first one is the main algorithm that the paper aims to propose and the second algorithm is an application of MFFs.

\subsection{Algorithm 1: meta fuzzy functions}

There are three components in the main algorithm that needs to have further clarification. The first one is the clarification of "function". A function consists of the combination of the methods. When we say "a function" we mean "a cluster" in FCM. The second one is the weights of the methods in functions. Weights of the methods are simply obtained from the degrees of memberships of a method in a cluster. The third one is the best meta fuzzy function $\left(M F F_{\text {best }}\right)$. Because we have as many functions as the number of clusters, we are looking for a function that has the best evaluation criteria. In this case, the function that has the best evaluation criteria is called $M F F_{\text {best }}$ and future estimations or forecasts are calculated with $\left(M F F_{\text {best }}\right)$.

- Step 1. Determination the existing related methods for the problem (i.e forecasting) and training of the related methods.

- Step 1.1. Determine $m$ existing methods for the problem.
- Step 1.2. The dataset is divided into two sets; training and test datasets.

$X=\left[X_{i j}\right], i=1,2, \ldots, p ; j=1,2 . ., n$

$$
X_{\text {train }}=\left[X_{i j}\right], i=1,2, \ldots, p ; j=1,2 . ., \text { ntrain }
$$

$X_{\text {test }}=\left[X_{i j}\right], i=1,2, \ldots, p ; j=n$ train $+1, n$ train $+2, \ldots, n$

- Step 1.3. Train the related existing methods for a given dataset by using $X_{\text {train }}$.

- Step 1.4. Obtain the outcomes (i.e forecasts) by using the trained methods for the test dataset, $X_{\text {test }}$. The input matrix of the MFFs are the collection of outcomes of the trained methods.

$Z=\left[Z_{i j}\right], i=1,2, \ldots$, ntest $; j=1,2 \ldots, m$

where $Z_{i j}$ is the outcomes of the $i$ th data point for $j$ th method.

$$
Z=\left[\begin{array}{ccccc}
Z_{1,1} & Z_{1,2} & \cdot & \cdot & Z_{1, \text { ntest }} \\
Z_{2,1} & Z_{2,2} & \cdot & \cdot & Z_{2, \text { ntest }} \\
\cdot & \cdot & \cdot & \cdot & \cdot \\
\cdot & \cdot & \cdot & \cdot & \cdot \\
Z_{m, 1} & Z_{m, 2} & \cdot & \cdot & Z_{m, n t e s t}
\end{array}\right]
$$

- Step 2. The input matrix $Z$ is divided into two, training $\left(Z_{\text {train }}\right)$ and test $\left(Z_{\text {test }}\right)$ sets. The training set is used to determine the weights of the methods in functions and the test set is used to evaluate the performance of MFFs.

$$
\begin{gathered}
Z_{\text {train }}=\left[Z_{i j}\right], i=1,2, \ldots, \text { ntrain } 1 ; j=1,2 \ldots, m \\
Z_{\text {ntest }}=\left[Z_{i j}\right], i=\text { ntrain } 1, \text { ntrain } 1+1, \ldots, \text { ntest } ; j=1,2 \ldots, m
\end{gathered}
$$

- Step 3. Determining the weights of the methods in functions. The input matrix $Z_{\text {train }}$ is clustered by using FCM. The degrees of memberships in each cluster are used to determine the weights of the methods in functions. In this case, a cluster represents a function.

- Step 3.1. Determine the fuzziness index parameter, number of clusters and initial cluster centers.

- Step 3.2. Calculate the membership value with the formula

$$
\begin{aligned}
\mu_{i k} & =\left[\sum_{j=1}^{c}\left(\frac{d\left(z_{k}, v_{i}\right)}{d\left(z_{k}, v_{j}\right)}\right)^{\frac{2}{m-1}}\right]^{-1}, \\
i & =1,2, \ldots, c ; k=1,2, \ldots, n
\end{aligned}
$$

under the constraint; $\sum_{i=1}^{c} \mu_{i k}=1$, if $\mu_{i k}<\alpha-c u t$, then $\mu_{i k}$ value will be taken as zero. $Z$ is the input matrix, $v$ is the cluster centers, $d($.) stands for Euclidean distance function, $c$ is the number of clusters, and $m$ is the fuzziness parameter in Eqs. (7)-(8).

- Step 3.3. Calculate the new cluster centers.

$$
v_{i}=\frac{\sum_{k=1}^{n} \mu_{i k}^{m} z_{k}}{\sum_{k=1}^{n} \mu_{i k}^{m}}
$$

- Step 3.4. Repeat Step 2 and Step 3 until the difference of clusters between two iterations drops under some threshold or the number of iterations is reached. 
- Step 4. Obtaining the meta fuzzy functions.

MFFs, which are given in Eq. (9), are obtained by using the degrees of memberships that are calculated for the training set.

$\operatorname{MFF}_{i}(z)=\sum_{j=1}^{m} w_{i j} z_{j}, i=1,2, \ldots, c$

$w_{i j}=\frac{\mu_{i j}}{\sum_{j=1}^{m} \mu_{i j}}, i=1,2, \ldots, c$

where $M F F_{i}$ stands for the $i$ th meta fuzzy function, $\mu_{i j}$ stands for the degree of membership value of the $j$ th method in $i$ th cluster and c represents the number of clusters.

- Step 5. Repeat Step 3-4 for different $m$ and $c$.

- Step 6. Selecting the best combination of the methods in a function.

The function that has the best evaluation criteria is chosen as the best meta fuzzy function $\left(M F F_{\text {best }}\right)$.

- Step 7. The outcomes of the MFFs' system is calculated by using the $M F F_{\text {best }}$ for the test set, $Z_{\text {test }}$.

$F=M F F_{\text {best }}\left(z_{\text {test }}\right)$

where $F$ is the outcomes of the MFFs.

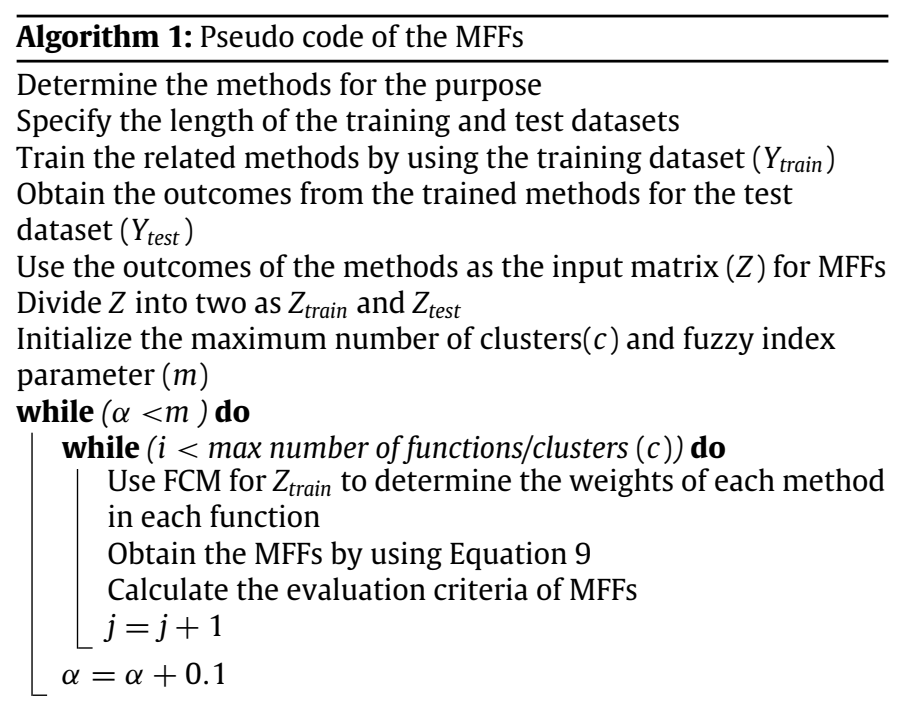

Return the best function ( $M F F_{\text {best }}$ ) that has the best evaluation criteria

Calculate the outcomes $(F)$ by using $M F F_{\text {best }}$

\subsection{Algorithm 2: MFFs of R-T1FFs}

Because fuzzy set theory deals with the uncertainty, there have been many studies employing fuzzy set theory to forecasting problems. Some of these studies are conducted by Song and Chissom [11,12], Chen [13] Chen and Zhang [14], Gupta et al. [15], and Aladag et al. [16]. In the literature, it has been also shown that the combination of methods improves the forecasting performances. Makridakis [17] has pointed out that combining reduces the variance of forecasting errors. Thus, the empirical finding of combining improves forecasting accuracy holds true. Bates and Granger [18] combined two forecasting methods in their study and got better forecasting performances than both forecasting methods. Granger and Ramanathan [19] proposed 3 approaches for combining three forecasting methods. Besides, some of the studies on combining forecasts are conducted by Bunn [20], Newbold and
Granger [21], Zou and Yang [22], and Aladag et al. [23]. In this application, the models of a recently introduced time series forecasting method (R-T1FFs) are aggregated with MFFs. Aggregating the different forecasting methods is left for future work.

The important issue with recurrent systems is stability. R-T1FFs are very sensitive the initial starting points and different parameter specifications as well. Therefore, the model that has the best evaluation criteria is searched iteratively with different starting points and parameter specifications to ensure stability in R-T1FFs. In, R-T1FFs, there are 3 parameters, which has an effect on the forecasting performances, to be adjusted; the number of clusters, the number of lags for $\mathrm{AR}(\mathrm{p})$ model, and the number of lags for MA(q) model. The pseudo code of the R-T1FFs based on MFFs is given Algorithm 2.

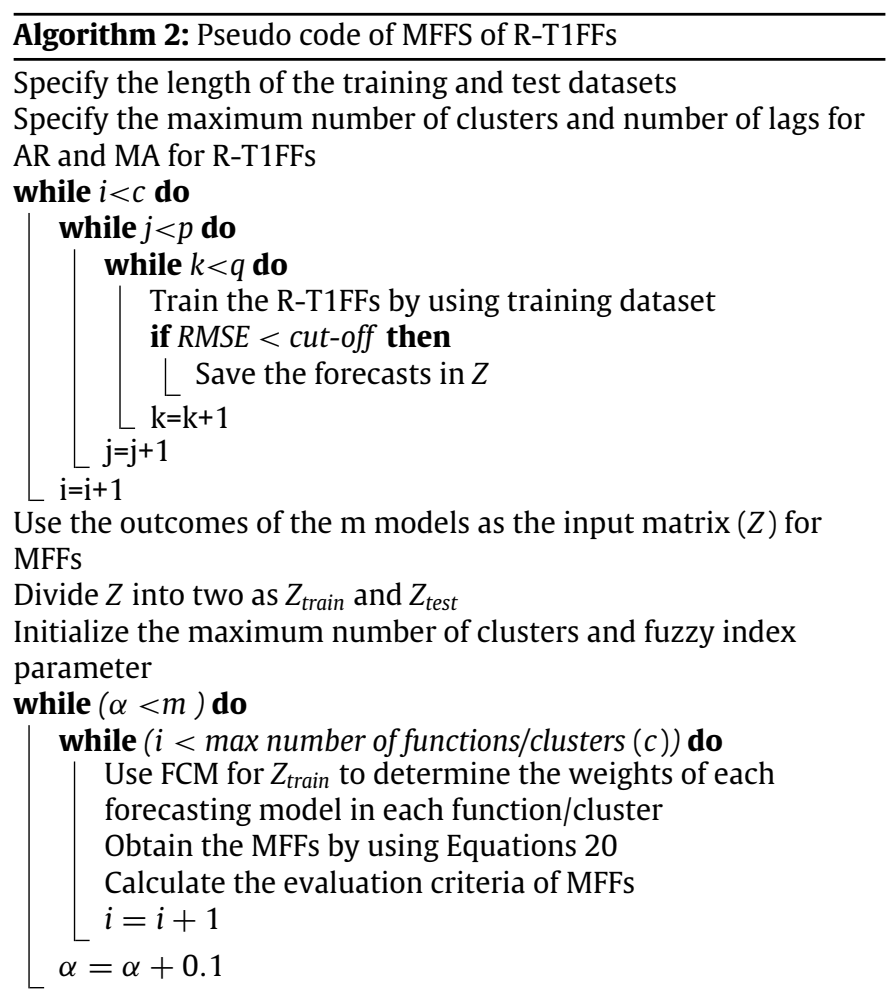

Return the function $\left(M F F_{\text {best }}\right)$ that has the best evaluation criteria Calculate the forecasts $(F)$ by using $M F F_{\text {best }}$ for $Z_{\text {test }}$

- Step 1. The time series dataset is divided into two as training and test datasets by using the block partitioning technique.

$Y=\left[Y_{i}\right], i=1,2, \ldots, t$

$Y_{\text {train }}=\left[Y_{i}\right], i=1,2 . .$, ntrain

$Y_{\text {test }}=\left[Y_{i}\right], i=n$ train +1, ntrain $+2, \ldots, n$

- Step 2. Obtaining the input matrix of MFFs from R-T1FFs.

- Step 2.1. There are 3 parameters, which have effect on forecasting performances, to be adjusted for R-T1FFs, the number of clusters and the number of lags for AR and MA for R-T1FFs forecasting approach.

- Step 2.2. Obtain $m$ different forecasting models from R-T1FFs with different parameter specifications for $Y_{\text {train }}$ by putting a restriction on RMSE value. R-T1FFs is a forecasting method that has been recently introduced. The detailed steps of R-T1FFs are found in [4]. 
- Step 2.3. Obtain the forecasts of $m$ models The input matrix $(Z)$ are constituted of the forecasts of the $m$ models for MFFs.

$Z=\left[Z_{i j}\right], i=1,2, \ldots$, ntest $; j=1,2, \ldots, m$

Step 1-2 are proceeded to determine the input matrix of the MFFs. In this case, because we have a forecasting problem, the models of R-T1FFs with different parameter specifications are aggregated in functions. The rest of the algorithm is the main algorithm of MFFs but modified for the forecasting problem.

- Step 3. The input matrix $Z$ is divided into two, training $\left(Z_{\text {train }}\right)$ and test $\left(Z_{\text {test }}\right)$ sets. The training set is used to determine the weights of the models of R1TFFs in functions and the test set is used to evaluate the performance of MFFs.

$Z_{\text {train }}=\left[Z_{i j}\right], i=1,2, \ldots, n$ train $1 ; j=1,2 \ldots, m$

$Z_{\text {ntest }}=\left[Z_{i j}\right], i=n$ train $1, n$ train $1+1, \ldots, n$ test $; j=1,2 \ldots, m$

- Step 4. Initialize the maximum number of clusters $(c)$ and fuzziness parameter $(m)$. There are some studies in the literature on specifying the fuzziness parameter.

Many studies in literature have been conducted by researchers based on the selection of fuzziness parameter. One of them is introduced by Pal and Bezdek [24], which points out that the optimal value of $m$ is limited to [1.5,2.5]. On the other hand, Ozkan and Turksen [25] identified that the upper and lower value of $m$ is 1.4 and 2.6, respectively. Chan and Cheung [26] suggested that the value of $m$ should be in $[1.25,1.75]$ in a study of word recognition. Also, Bezdek [27] proposed that the optimum selection of $m$ is 2 .

- Step 5. The weights of each model in each function is obtained by using the degree of memberships values in FCM.

- Step 5.1. Determine the fuzziness index parameter, number of clusters and initial cluster centers.

- Step 5.2. Calculate the membership value with the formula

$$
\begin{aligned}
\mu_{i k} & =\left[\sum_{j=1}^{c}\left(\frac{d\left(z_{k}, v_{i}\right)}{d\left(z_{k}, v_{j}\right)}\right)^{\frac{2}{m-1}}\right]^{-1}, \\
i & =1,2, \ldots, c ; k=1,2, \ldots, n
\end{aligned}
$$

under the constraint; $\sum_{i=1}^{c} \mu_{i k}=1$, if $\mu_{i k}<\alpha-c u t$, then $\mu_{i k}$ value will be taken as zero. $Z$ is the input matrix, $v$ is the cluster centers, $d($.) stands for Euclidean distance function, $c$ is the number of clusters, and $m$ is the fuzziness parameter in Eqs. (1)-(2).

- Step 5.3. Calculate the new cluster centers.

$$
v_{i}=\frac{\sum_{k=1}^{n} \mu_{i k}^{m} z_{k}}{\sum_{k=1}^{n} \mu_{i k}^{m}}
$$

- Step 5.4. Repeat Step 2 and Step 3 until the difference of clusters between two iterations drops under some threshold or the number of iterations is reached.

- Step 6. Using the degree of memberships values and the forecasts that are obtained from different models, MFFs are calculated with the formula given in Eq. (6).

$\operatorname{MFF}_{i}(z)=\sum_{j=1}^{m} w_{i j} z_{j}, i=1,2, \ldots, c$

$$
w_{i j}=\frac{\mu_{i j}}{\sum_{j=1}^{m} \mu_{i j}}, i=1,2, \ldots, c
$$

where MFF stands for the meta fuzzy function and c stands for the number of clusters.

- Step 7. Step 6 is repeated for the number of clusters times.

- Step 8. Step 5-7 is repeated for different $m$ and $c$ specifications.

- Step 9. The function that has the minimum Root Mean Squared Errors (RMSE) or Mean Absolute Percentage Errors (MAPE) is selected as the $M F F_{\text {best }}$.

- Step 10. The forecast are calculated by using $M F F_{\text {best }}$.

$F=M_{\text {best }}\left(z_{\text {test }}\right)$

where $\mathrm{F}$ is the forecasts of the MFFs.

\section{Evaluation}

9 real world time series datasets were analyzed to evaluate the performance of the MFFs of R-T1FFs. All calculations are done using $\mathrm{R}$, a statistical programming language. The first dataset is the Australian Beer Consumption (ABC) dataset [28] which were observed for each quarter from 1956 to 1994 . The next 4 datasets are from the Istanbul Stock Exchange (BIST100) [29]. The observations of four datasets were observed daily for the first half of a year from 2009 to 2012. The last 4 datasets are from Dow Jones stock exchange. The observations of the Dow Jones index are daily measured year by year from 2010 to 2013. The summary of the datasets and parameter selection criteria of R-T1FFs are given Table 1. The methods are evaluated using MAPE and RMSE. RMSE and MAPE values are two commonly used evaluation metric in the literature for forecasting purposes. RMSE aims to measure the magnitude of the error. It uses the actual and predicted values of a time series in a distance function to measure. MAPE, as well, uses predicted and actual values of a time series to measure the average magnitude of the errors. Both error measures, aim to obtain the average model forecasting error in observations of a time series and both criteria range between 0 and infinity. The lower RMSE/MAPE value means the better forecasting accuracy for both metric. The formulations of RMSE and MAPE values are given in Eqs. (23)-(24)

$R M S E=\sqrt{\frac{1}{n} \sum_{t=1}^{n}\left(x_{t}-\widehat{x}_{t}\right)^{2}}$

$M A P E=\frac{1}{n} \sum_{t=1}^{n}\left|\frac{x_{t}-\widehat{x_{t}}}{x_{t}}\right|$

where $x_{t}$ is the actual values of the time series and $\widehat{x}_{t}$ is the forecasts.

The stock index data are known as a complex time series because there are many factors that can affect stock prices. Because of mostly non-linear structure of stock index datasets, statistical approaches usually fail to give accurate results. Therefore, nonstatistical approaches commonly use stock index datasets for the evaluation of their proposed methods. IEX and DowJones datasets are chosen in this sense and to be able to compare the forecasting results of MFFs of R-T1FFs with the existing methods that used the same datasets previously. $\mathrm{ABC}$ is another commonly used dataset by researchers and it contains both seasonality and trend. Therefore, ABC, IEX, and DowJones datasets are chosen to be able to compare the forecasting accuracy of the MFFs of R-T1FFs with the existing ones.

In order to perform MFFs, R-T1FFs is chosen as an application method. In other words, different models that are obtained from 
Table 1

Summary of the datasets and parameter selection criteria of R-T1FFs.

\begin{tabular}{lllllll}
\hline & Series/Year & Obs. & AR & MA & Clusters numbers & ntest \\
\hline 1 & ABC & 147 & $1-10$ & $1-2$ & $2-10$ & 16 \\
2 & BIST100/2009 & 103 & $1-5$ & $1-2$ & $2-5$ & 15 \\
3 & BIST100/2010 & 104 & $1-5$ & $1-2$ & $2-5$ & 15 \\
4 & BIST100/2011 & 106 & $1-5$ & $1-2$ & $2-5$ & 15 \\
5 & BIST100/2012 & 106 & $1-5$ & $1-2$ & $2-5$ & 15 \\
6 & DowJones/2010 & 252 & $1-5$ & $1-2$ & $2-5$ & 10 \\
7 & DowJones/2011 & 251 & $1-5$ & $1-2$ & $2-5$ & 10 \\
8 & DowJones/2012 & 250 & $1-5$ & $1-2$ & $2-5$ & 10 \\
9 & DowJones/2013 & 252 & $1-5$ & $1-2$ & $2-5$ & 10 \\
\hline
\end{tabular}

R-T1FFs will be aggregated with MFFs. R-T1FFs is a recently introduced forecasting method by Tak et al. [4]. There are 3 parameters to be adjusted for R-T1FFs, the number of lags for $\operatorname{AR}(p)$ and $\operatorname{MA}(q)$ and the number of clusters, $c$. Although there are numerous studies on determining the optimum number of clusters for FCM, there is no consensus on which one the best is. Therefore, rather than using a method for determining the optimum number of clusters, the one that gives the minimum SSEs is chosen as the optimum number of clusters for R-T1FFs.

There are many information criteria for selecting the optimum lag length for ARMA model; root mean squares (RMSE), Schwartz information criteria (SIC), Hannan-Quinn criterion (HQ), Akaike information criteria (AIC), and etc. There is no consensus on the question of "which criteria the best is to determine the optimum number of lags". Thus, one approach to this is to use all criteria cited above and chose the one that has the smallest lag length. However, in R-T1FFs approach, sum of squares errors is used to determine the number of lags for AR and MA models. The optimum numbers of lags for AR and MA are searched iteratively and the ones that have the smallest SSEs are chosen. However, in the evaluation part of the proposed method, we are not looking for the best sets of lags for AR and MA models, we are randomly selecting models with different parameter specifications because we are aggregating numerous models that are obtained with different sets of lags lengths and the cluster numbers. Optimum numbers of $p, q$, and $c$, which gives the minimum SSEs, are searched iteratively in R-T1FFs. For each dataset, some cut-off (RMSE/MAPE) value is determined and 10 models for each dataset under some RMSE/MAPE value is stored. The one that has the best RMSE/MAPE, the one that has the worst RMSE/MAPE value, and randomly 8 models are selected from the storage as an input matrix $(Z)$ for MFFs.

There are two parameters to be adjusted in MFFs; the fuzziness parameter $(m)$ and the number of clusters $(c)$. The fuzziness parameter is varied from 1.3 and 3 with an increase rate of 0.1 and
AUST Dataset

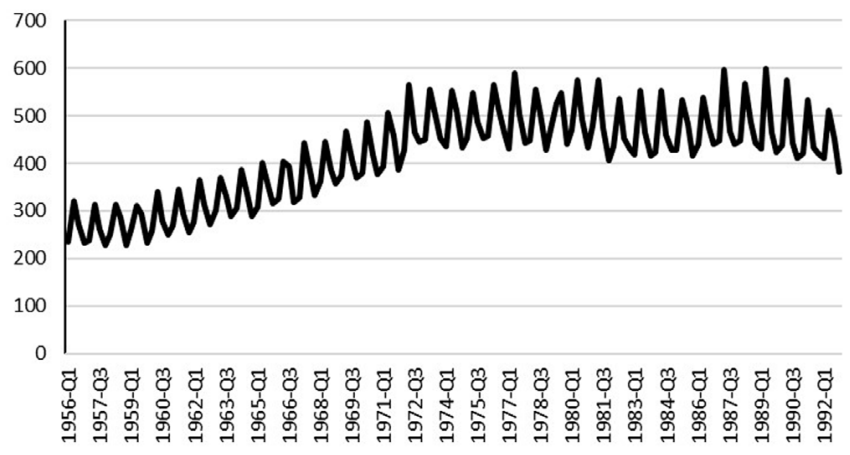

Fig. 1. Line plot of $A B C$ dataset.

the number of clusters is varied from 2 to 5 . The $m$ and $c$ which give the best outcomes, in terms of RMSE/MAPE, are selected for MFFs.

\subsection{ABC dataset}

In the first evaluation, the $\mathrm{ABC}$ dataset was used. This dataset consists of 148 observations that were observed quarterly from 1956 to 1994. The line plot of the ABC dataset is given in Fig. 1.

In the training phase of the R-T1FFs, the last 16 observations were left for the test set $\left(Y_{\text {test }}\right)$ for ABC and 10 models were obtained with different parameter $(p, q, c)$ specifications. 16 data points are forecasted by using trained models and given in Table 2. In order to obtain MFFs and MFF best, the first half of 16 forecasts of the models are used as training set, $Z_{\text {train }}$ (see Table 3 ). The last 8 observations $\left(Z_{\text {test }}\right)$ (see Table 4 ) are used to be able to compare the performance of the proposed method with the existing methods. Winter's multiplicative exponential smoothing (WMES), SARIMA [30], Feed-Forward Artificial Neural Network (FFANN), Adaptive-Network-based Fuzzy Inference Systems (ANFIS) [31], Modified ANFIS (MANFIS) [32], Autoregressive ANFIS (AR-ANFIS) [33], and R-T1FF [4], which used the ABC datasets as applications previously, are selected to compare the performance of MFFs and these results are quoted from [4].

The algorithm searched for the best models when the number of clusters is varied from 2 to 5 and the fuzziness parameter is varied from 1.3 to 3 with the increase rate of 0.1 for $A B C$ dataset. Under these conditions, the function which has the minimum RMSE value is obtained when the number of clusters (functions) is 3 and the fuzziness parameter is 1.4 . The weights of the models, which are

Table 2

10 different forecasting results of the R-T1FF for the ABC dataset.

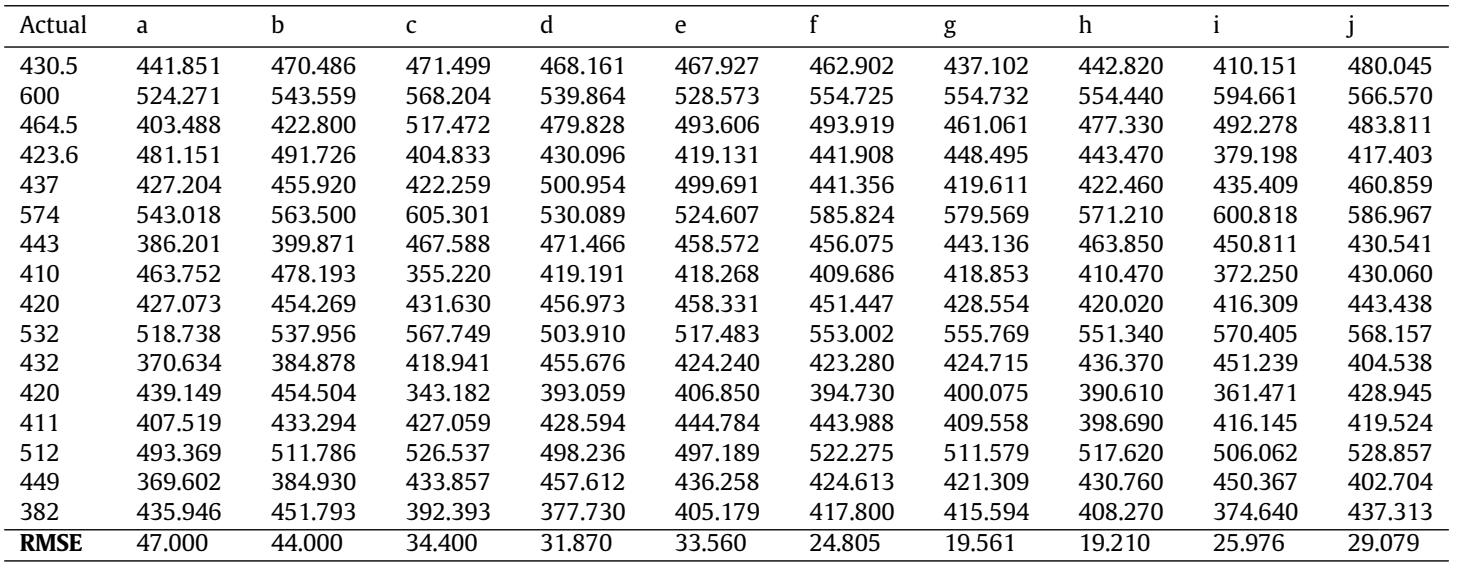


Table 3

Training dataset $\left(Z_{\text {train }}\right)$ for MFFs.

\begin{tabular}{|c|c|c|c|c|c|c|c|c|c|c|}
\hline Actual & $a$ & b & c & d & e & $\mathrm{f}$ & $\mathrm{g}$ & $\mathrm{h}$ & $\mathrm{i}$ & $\mathrm{j}$ \\
\hline 430.5 & 441.851 & 470.486 & 471.499 & 468.161 & 467.927 & 462.902 & 437.102 & 442.820 & 410.151 & 480.045 \\
\hline 600 & 524.271 & 543.559 & 568.204 & 539.864 & 528.573 & 554.725 & 554.732 & 554.440 & 594.661 & 566.570 \\
\hline 464.5 & 403.488 & 422.800 & 517.472 & 479.828 & 493.606 & 493.919 & 461.061 & 477.330 & 492.278 & 483.811 \\
\hline 437 & 427.204 & 455.920 & 422.259 & 500.954 & 499.691 & 441.356 & 419.611 & 422.460 & 435.409 & 460.859 \\
\hline 574 & 543.018 & 563.500 & 605.301 & 530.089 & 524.607 & 585.824 & 579.569 & 571.210 & 600.818 & 586.967 \\
\hline 443 & 386.201 & 399.871 & 467.588 & 471.466 & 458.572 & 456.075 & 443.136 & 463.850 & 450.811 & 430.541 \\
\hline
\end{tabular}

Table 4

Training dataset $\left(Z_{\text {test }}\right)$ for MFFs.

\begin{tabular}{|c|c|c|c|c|c|c|c|c|c|c|}
\hline Actual & $a$ & $\mathrm{~b}$ & c & $d$ & e & $f$ & $g$ & h & i & $\mathrm{j}$ \\
\hline 420 & 427.073 & 454.269 & 431.630 & 456.973 & 458.331 & 451.447 & 428.554 & 420.020 & 416.309 & 443.438 \\
\hline 532 & 518.738 & 537.956 & 567.749 & 503.910 & 517.483 & 553.002 & 555.769 & 551.340 & 570.405 & 568.157 \\
\hline 432 & 370.634 & 384.878 & 418.941 & 455.676 & 424.240 & 423.280 & 424.715 & 436.370 & 451.239 & 404.538 \\
\hline 411 & 407.519 & 433.294 & 427.059 & 428.594 & 444.784 & 443.988 & 409.558 & 398.690 & 416.145 & 419.524 \\
\hline 512 & 493.369 & 511.786 & 526.537 & 498.236 & 497.189 & 522.275 & 511.579 & 517.620 & 506.062 & 528.857 \\
\hline 449 & 369.602 & 384.930 & 433.857 & 457.612 & 436.258 & 424.613 & 421.309 & 430.760 & 450.367 & 402.704 \\
\hline RMSE & 41.731 & 41.973 & 32.193 & 22.455 & 22.286 & 25.531 & 19.319 & 17.546 & 25.986 & 32.114 \\
\hline MAPE & 0.074 & 0.083 & 0.0546 & 0.044 & 0.046 & 0.055 & 0.0354 & 0.0333 & 0.0389 & 0.0638 \\
\hline
\end{tabular}

Table 5

The weights of the models in functions and RMSE values of the functions for $Z_{\text {train }}$.

\begin{tabular}{llll}
\hline Model & $M F F_{1}$ & $M F F_{2}$ & $M F F_{3}$ \\
\hline a & 0.496 & 0.000 & 0.000 \\
b & 0.495 & 0.000 & 0.000 \\
c & 0.000 & 0.476 & 0.000 \\
d & 0.000 & 0.000 & 0.174 \\
e & 0.000 & 0.000 & 0.171 \\
f & 0.000 & 0.000 & 0.176 \\
g & 0.000 & 0.000 & 0.145 \\
h & 0.000 & 0.000 & 0.164 \\
i & 0.001 & 0.495 & 0.002 \\
j & 0.008 & 0.029 & 0.166 \\
\hline RMSE & 47.452 & 27.484 & $24.023^{*}$ \\
MAPE & 0.091 & 0.053 & $0.041^{*}$ \\
\hline
\end{tabular}

Table 6

Forecasts of MFFs for ABC dataset and RMSE and MAPE values of the functions.

\begin{tabular}{clll}
\hline Test & $M F F_{1}$ & $M F F_{2}$ & $M F F_{3}$ \\
\hline 9 & 440.67 & 424.39 & 443.69 \\
10 & 528.69 & 569.08 & 541.04 \\
11 & 378.01 & 434.51 & 428.40 \\
12 & 446.62 & 354.75 & 402.23 \\
13 & 420.39 & 421.43 & 424.86 \\
14 & 502.78 & 516.47 & 512.52 \\
15 & 377.51 & 441.12 & 429.30 \\
16 & 443.77 & 384.92 & 409.91 \\
\hline RMSE & 40.56 & 27.06 & $17.07^{*}$ \\
MAPE & 0.0757 & 0.0376 & $0.0344^{*}$ \\
\hline
\end{tabular}

calculated with Eq. (21), in functions and the RMSE and MAPE values of the functions for $Z_{\text {train }}$ are given in Table 5 .

Table 5 reveals that the best MFF is the third function in terms of RMSE and MAPE values. Therefore, forecasts are calculated by using $M F F_{\text {best }}=M F F_{3} . M F F_{\text {best }}$ is given in Eq. (25) and the forecasting results of $M F F_{\text {best }}$ and the other functions are given in Table 6.

$M F F_{\text {best }}=M \mathrm{MF}_{3}=0.174 * d+\cdots+0.102 * i+0.166 * j$

In order to evaluate the performance of the proposed method with the existing methods Table 7 is given. In terms of RMSE and MAPE values, it is obvious that the best forecasting results are obtained from the proposed method.

\subsection{BIST100 datasets}

There are 4 datasets year by year from 2009 to 2012 for BIST100. To obtain 10 models from R-T1FF, the number of clusters was varied from 2 to 5 , the AR lag was varied from 1 to 5 , and the MA lag was varied from 1 to 2 . The last 15 observations for all BIST100 datasets are selected as the test dataset $\left(Y_{\text {test }}\right)$. The input matrix $(Z)$ of MFFs are obtained from the 15 point forecasts of 10 models. The first 8 observations of $Z$ is selected as the training matrix $\left(Z_{\text {train }}\right)$ in order to determine the weights of the models in functions and $M F F_{\text {best }}$. The last 7 observations are left for test dataset $\left(Z_{\text {test }}\right)$ to evaluate the performance of the proposed method with the existing methods. ARIMA [30], Exponential Smoothing (ES) [34], Multilayer Perceptron ANN (MLP-ANN), Fuzzy Functions (FF) [5], Fuzzy Time Series Network (FTS-N) [35], and R-T1FF [4], which used the BIST100 datasets as applications previously, are selected to compare the performance of MFFs.

For MFFs, there are two parameters to be adjusted; fuzziness value $(m)$ and the number of functions $(c)$. The number of functions is varied from 2 to 5 and the fuzziness value is varied from 1.3 to 3 with the increase rate of 0.1 . The detailed results are given for the datasets of 2009 for BIST100. Only the input matrices and formal comparative tables are given for the rest of the datasets of BIST100.

\subsubsection{9}

For BIST100 dataset in 2009, 15 forecasts of 10 models are used as the input matrix, $Z$. The first 8 observations of $Z$ is used as training matrix $\left(Z_{\text {train }}\right)$ and the last 7 observations are used as the test dataset $\left(Z_{\text {test }}\right)$ and they are given in Tables 8 and 9, respectively.

The best MFFs are obtained when $m=2.4$ and $c=3$. The weight matrix that are calculated by FCM for $Z_{\text {train }}$ is given in Table 3.

The best function is determined from MAPE values of the MFFs for $Z_{\text {train }}$. In this case, $M F F_{\text {best }}$ is selected as $M F F_{2}$ (see Table 10). Thus, the forecasts of the proposed methods is obtained by using $M F F_{b e s t}$. $M F F_{\text {best }}$ is given in Eq. (26) and the corresponding forecasting results and the other functions are given in Table 11.

$M F F_{\text {best }}=M \mathrm{MF}_{2}=0.04 * a+\cdots+0.20 * i+0.21 * j$

6 existing forecasting methods are compared with the proposed method in Table 12 . It is obvious that the $M F F_{\text {best }}$ of the proposed 
Table 7

Forecasting results and RMSE/MAPE values of existing methods and MFF $_{\text {best }}$.

\begin{tabular}{|c|c|c|c|c|c|c|c|c|}
\hline Test & WMES & SARIMA & FFANN & ANFIS & MANFIS & AR-ANFIS & T1FF & $M F F_{\text {best }}$ \\
\hline 420 & 465.4 & 461.01 & 448.87 & 445.0127 & 430.31 & 419.428 & 431.60 & 443.69 \\
\hline 532 & 589.74 & 588.96 & 560.04 & 562.94 & 565.18 & 570.378 & 559.41 & 541.04 \\
\hline 420 & 455.89 & 454.64 & 408.64 & 416.16 & 392.14 & 400.06 & 394.99 & 402.23 \\
\hline 411 & 471.15 & 465.46 & 428.11 & 431.70 & 419.33 & 413.925 & 409.72 & 424.86 \\
\hline 512 & 597 & 594.71 & 537.70 & 544.98 & 536.88 & 549.318 & 525.60 & 512.52 \\
\hline RMSE & 67.032 & 60.06 & 23.79 & 26.95 & 21.47 & 23.44 & 18.30 & $17.07^{*}$ \\
\hline MAPE & 0.147 & 0.13 & 0.050 & 0.054 & 0.0424 & 0.040 & 0.036 & $0.034^{*}$ \\
\hline
\end{tabular}

Table 8

Training dataset $\left(Z_{\text {train }}\right)$ for 2009 .

\begin{tabular}{|c|c|c|c|c|c|c|c|c|c|c|}
\hline Test & $a$ & b & c & d & $\mathrm{e}$ & f & $\mathrm{g}$ & h & $\mathrm{i}$ & $\mathrm{j}$ \\
\hline 32806 & 32664.29 & 33398.2 & 32898 & 33420.2 & 33072.6 & 33200.3 & 33170.4 & 33132.9 & 33731.4 & 33269.3 \\
\hline 32203 & 32594.61 & 32876.5 & 32822.2 & 33109.5 & 32938.4 & 32647.9 & 32708 & 32933.5 & 32558.4 & 32121.6 \\
\hline 32829 & 32786.4 & 32774.8 & 33050.5 & 32815.8 & 33119.5 & 32544.1 & 33142.7 & 33207.1 & 32678 & 32648.3 \\
\hline 33095 & 33133.76 & 32884 & 32897.1 & 33230.3 & 32837.1 & 33271 & 33245.2 & 33012.1 & 33769.5 & 33187.7 \\
\hline 33485 & 33064.32 & 33037.2 & 33188.1 & 33250.8 & 33252.2 & 33230.5 & 33446.1 & 33274.8 & 33622.9 & 33411.3 \\
\hline
\end{tabular}

Table 9

Test dataset $\left(Z_{\text {test }}\right)$ for 2009 .

\begin{tabular}{|c|c|c|c|c|c|c|c|c|c|c|}
\hline Test & $\mathrm{a}$ & b & c & d & e & f & g & $\mathrm{h}$ & $\mathrm{i}$ & $\mathrm{j}$ \\
\hline 34721 & 35364.49 & 35047.95 & 34687.79 & 34455.7 & 35052.9 & 34458.45 & 35196.19 & 34352.33 & 35180.2 & 34534.4 \\
\hline 35015 & 35261.99 & 34516.6 & 34836.9 & 34992.4 & 34797.1 & 35348.2 & 34882.9 & 34902 & 35495.7 & 34964.2 \\
\hline 35408 & 35335.15 & 35346 & 35112 & 35140 & 35117.1 & 35408.5 & 35411.1 & 35201.7 & 35026.4 & 35076.3 \\
\hline 35169 & 35317.37 & 34982 & 34991.2 & 34751 & 34923.5 & 35468.3 & 34821.5 & 35042.8 & 34799.2 & 34739.5 \\
\hline 35021 & 35280.65 & 35108.8 & 35292.7 & 34896.7 & 35244.3 & 35387.4 & 35169.4 & 35354.5 & 34849.2 & 34420.1 \\
\hline 35003 & 34997.85 & 35075.1 & 35148.9 & 34808.7 & 35106.8 & 35228.2 & 34968.7 & 35203.9 & 35200.3 & 34648 \\
\hline MAPE & 0.0095 & 0.0108 & 0.00683 & 0.00739 & 0.0087 & 0.0097 & 0.00935 & 0.0093 & 0.00861 & 0.00487 \\
\hline
\end{tabular}

Table 10

Weights of the models in functions and RMSE values of the functions for $Z_{\text {train }}$.

\begin{tabular}{llll}
\hline Model & $M F F_{1}$ & $M F F_{2}$ & $M F F_{3}$ \\
\hline a & 0.04 & 0.04 & 0.28 \\
$\mathrm{~b}$ & 0.06 & 0.06 & 0.22 \\
$\mathrm{c}$ & 0.12 & 0.05 & 0.14 \\
$\mathrm{~d}$ & 0.11 & 0.13 & 0.06 \\
$\mathrm{e}$ & 0.18 & 0.04 & 0.05 \\
$\mathrm{f}$ & 0.07 & 0.18 & 0.06 \\
$\mathrm{~g}$ & 0.12 & 0.09 & 0.08 \\
$\mathrm{~h}$ & 0.21 & 0.00 & 0.00 \\
$\mathrm{i}$ & 0.05 & 0.20 & 0.06 \\
$\mathrm{j}$ & 0.05 & 0.21 & 0.06 \\
\hline RMSE & 575.64 & $531.48^{*}$ & 637.11 \\
MAPE & 0.012 & $0.011^{*}$ & 0.013 \\
\hline
\end{tabular}

methods outperforms other methods in terms of both RMSE and MAPE values.
Table 11

Forecasting results and RMSE/MAPE values of the functions.

\begin{tabular}{clll}
\hline Model & $M F F_{1}$ & $M F F_{2}$ & $M F F_{3}$ \\
\hline 9 & 35084.72 & 34740.53 & 34865.10 \\
10 & 34937.89 & 35112.87 & 34974.52 \\
11 & 35205.86 & 35195.13 & 35262.91 \\
12 & 35373.75 & 35112.96 & 35169.56 \\
13 & 34964.67 & 34949.34 & 35051.79 \\
14 & 35166.00 & 34961.31 & 35142.70 \\
15 & 35067.51 & 34996.57 & 35032.74 \\
\hline RMSE & 269.64 & $156.12^{*}$ & 154.96 \\
MAPE & 0.0064 & $0.0035^{*}$ & 0.0037 \\
\hline
\end{tabular}

3.2.2. 2010

The 15 forecasts of the 10 models are used as the input matrix $(Z)$ of MFFs. The input matrix is divided into two training $\left(Z_{\text {train }}\right)$ and test $\left(Z_{\text {test }}\right)$ sets. Tables $13-14$ represents the training and test sets respectively for 2010 .

Table 12

Forecasting results and RMSE/MAPE values of existing methods and $M F F_{\text {best }}$.

\begin{tabular}{llllllll}
\hline Test & ARIMA & ES & MLP-ANN & FF & FTS-N & R-T1FF & MFF best \\
\hline 34721 & 35140 & 35139.7 & 34926.15 & 35353.47 & 34559.35 & 34676.64 & 34740.53 \\
35015 & 34721 & 34720.55 & 34547.22 & 35065.66 & 34999.72 & 35140.3 & 35112.87 \\
35408 & 35015 & 35014.5 & 34887.24 & 35247.61 & 35042.18 & 35396.82 & 35195.13 \\
34861 & 35408 & 35407.68 & 35108.24 & 35720.93 & 35257.93 & 35186.02 & 35112.96 \\
35169 & 34861 & 34860.56 & 34727.41 & 35196.88 & 35186.01 & 34850.95 & 34949.34 \\
35021 & 35169 & 35168.73 & 35002.28 & 35444.51 & 35103.95 & 34719.4 \\
35003 & 35021 & 35021.01 & 34844.72 & 35369.76 & 35103.37 & 34799.38 \\
\hline RMSE & 344.9114 & 344.9628 & 340.929 & 460.1925 & 218.7582 & 225.8351 & 34961.31 \\
MAPE & 0.0087 & 0.0087 & 0.0084 & 0.0103 & 0.0046 & 0.0054 \\
\hline
\end{tabular}


Table 13

Training dataset $\left(Z_{\text {train }}\right)$ for 2010 .

\begin{tabular}{|c|c|c|c|c|c|c|c|c|c|c|}
\hline Test & $\mathrm{a}$ & b & c & $\mathrm{d}$ & $\mathrm{e}$ & f & g & h & $\mathrm{i}$ & $\mathrm{j}$ \\
\hline 56448 & 55203.7 & 54002.99 & 54505.2 & 54651.9 & 55712.9 & 54246 & 55156.3 & 54278.5 & 54508.2 & 53646.6 \\
\hline 56462 & 55430.2 & 57199.94 & 55530.5 & 56039.4 & 54702.9 & 56567.6 & 56171.6 & 56015.6 & 55216.4 & 55535.8 \\
\hline 57976 & 55649.8 & 56313.11 & 56206.9 & 55434.7 & 54892.8 & 56192.2 & 55902.7 & 56263.6 & 55566.1 & 56928.2 \\
\hline 57930 & 56654.4 & 57872.39 & 57369.6 & 58269 & 55501.2 & 56901.2 & 56574.6 & 57591.5 & 56468.4 & 57810.5 \\
\hline 55748 & 57159.9 & 57853.95 & 57775.3 & 57546.6 & 56517.8 & 57313.8 & 56717.9 & 57859 & 57165.4 & 58141 \\
\hline 56071 & 57115.7 & 56787.94 & 56875 & 57289.3 & 57332.7 & 57030 & 56692.5 & 56863.6 & 56906.5 & 56457.9 \\
\hline 56978 & 56273.4 & 57608.18 & 56283.1 & 56847.7 & 56357.4 & 57235.6 & 56344.1 & 56190.2 & 56295 & 56074 \\
\hline
\end{tabular}

Table 14

Test dataset $\left(Z_{\text {test }}\right)$ for 2010

\begin{tabular}{|c|c|c|c|c|c|c|c|c|c|c|}
\hline Test & $a$ & b & c & d & e & $\mathrm{f}$ & g & h & $\mathrm{i}$ & $\mathrm{j}$ \\
\hline 54112 & 55002.7 & 54570.12 & 55544.2 & 55626.9 & 55155.3 & 55481.2 & 54994 & 54980.4 & 55435.2 & 55263 \\
\hline 54558 & 54175.8 & 55021.08 & 54683.5 & 55289.4 & 54194.5 & 55026.7 & 54385.5 & 54296.7 & 54655 & 54275 \\
\hline 52257 & 54000.3 & 54351.85 & 54665.1 & 53843.5 & 53601.7 & 54938.6 & 54223.6 & 54475.5 & 54457.1 & 54687.1 \\
\hline 54498 & 53165.3 & 54032.2 & 53818.7 & 52800 & 52716.4 & 54286.2 & 53144.3 & 53822.1 & 53560.2 & 53850.2 \\
\hline 55234 & 53692.7 & 54394.35 & 54294.3 & 54554.3 & 53315.4 & 54674.9 & 53360.7 & 54344.7 & 53956.1 & 54691.7 \\
\hline 54385 & 54329.8 & 56158.79 & 54798.3 & 55279.5 & 53932.1 & 55447.3 & 53879.2 & 55024 & 54522.4 & 55152.8 \\
\hline
\end{tabular}

Table 15

Forecasting results and RMSE/MAPE values of existing methods and $M F F_{\text {best }}$.

\begin{tabular}{|c|c|c|c|c|c|c|c|}
\hline Test & ARIMA & ES & MLP-ANN & $\mathrm{FF}$ & FTS-N & R-T1FF & $M F_{\text {best }}$ \\
\hline 54112 & 54450 & 54449.936 & 54397.950 & 53543.007 & 55431.528 & 52984.810 & 55207.13 \\
\hline 54558 & 54112 & 54111.607 & 54057.710 & 53788.681 & 54495.642 & 54419.000 & 54686.15 \\
\hline 52257 & 54558 & 54558.150 & 54507.230 & 54475.042 & 54629.554 & 53166.650 & 54358.55 \\
\hline 54104 & 52257 & 52257.121 & 52274.930 & 51697.376 & 53634.931 & 51463.420 & 53046.54 \\
\hline 54498 & 54104 & 54103.459 & 54049.690 & 54377.843 & 53584.444 & 54183.150 & 53577.29 \\
\hline 55234 & 54498 & 54497.941 & 54446.490 & 54445.135 & 53960.884 & 54071.150 & 54205.09 \\
\hline 54385 & 55234 & 55233.664 & 55194.750 & 55221.339 & 54360.633 & 55612.920 & 54989.81 \\
\hline MAPE & 0.0183 & 0.018 & 0.018 & 0.020 & $0.017^{*}$ & 0.020 & 0.018 \\
\hline
\end{tabular}

Table 16

Training dataset $\left(Z_{\text {train }}\right)$ for 2011 .

\begin{tabular}{|c|c|c|c|c|c|c|c|c|c|c|}
\hline Test & $\mathrm{a}$ & b & c & d & e & f & $g$ & $\mathrm{~h}$ & i & $\mathrm{j}$ \\
\hline 67260 & 67488.8 & 67664.6 & 67506.8 & 67118.3 & 67485.4 & 67862 & 67500.5 & 67782.3 & 67820.8 & 67553.7 \\
\hline 65643 & 66944.7 & 67173.5 & 66922.2 & 66669.3 & 66957.9 & 67384.6 & 66803.8 & 67268.4 & 67314.6 & 66610.2 \\
\hline 66535 & 65292.9 & 66070.5 & 65300.2 & 66316.6 & 65871.7 & 66432.9 & 65378 & 66282.9 & 66738.1 & 66147.6 \\
\hline 65418 & 64250.2 & 65068.7 & 64244.6 & 65036.5 & 65314.7 & 65326.4 & 64415.3 & 65195.1 & 65393.3 & 64185.8 \\
\hline 65385 & 65073.5 & 64589.6 & 65066.1 & 63952.1 & 65160.1 & 64957.5 & 65265.3 & 64819.6 & 64517.4 & 64940.6 \\
\hline 63733 & 65045.3 & 64822.4 & 65044.1 & 63921.9 & 65215.8 & 64941.2 & 64881 & 64859.6 & 64282.4 & 63355.5 \\
\hline
\end{tabular}

The minimum RMSE value is obtained when $m=2.5$ and $c=3$ for $Z_{\text {train }}$. The best function $\left(M F F_{\text {best }}\right)$ is determined as the first function that is given in Eq. (27).

$$
M F_{\text {best }}=M F_{1}=0.04 * a+0.14 * b+\cdots+0.08 * i+0.11 * j
$$

We are able to say that the performance of the proposed method is better than all existing forecasting methods in terms of RMSE value in Table 15.

\subsubsection{1}

There are 106 observations in BIST100 dataset for 2011 and the last 15 observations are taken as the test dataset. Using R-T1FF with different parameter specifications, 10 models are obtained. The first 8 observations of the 10 models are used as the training and the rest of the observations are use as the test dataset. Training and test sets are given in Tables 16-17, respectively.

The minimum RMSE value is obtained when $m=2.6$ and $c=4$. The weights of the models in functions are obtained by using the degree of membership values of the models in clusters for $Z_{\text {train }}$. The best function $\left(M F F_{b e s t}\right)$ is determined as the third meta fuzzy function and given in Eq. (28).

$M F F_{\text {best }}=M F F_{3}=0.06 * b+0.51 * d+\cdots+0.09 * i+0.18 * j(28)$

Comparing $M F F_{\text {best }}$ with the existing methods in Table 18, it is obvious that the best and second best forecasts are obtained from the proposed method in terms of RMSE and MAPE values, respectively.

\subsubsection{2}

The number of observations is 106 in BIST100 dataset for 2012. The forecasting results of 10 models of R-T1FFs method is given in Tables $19-20$ as training and test datasets, respectively.

The minimum RMSE value is obtained when $m=2$ and $c=3$. $M F F_{\text {best }}$ for 2012 is given in Eq. (29).

$M F F_{\text {best }}=M F F_{3}=0.04 * i+0.96 * j$

Comparing $M F F_{\text {best }}$ with the existing methods in Table 21, it is obvious that the best and second best forecasts are obtained from the proposed method in terms of MAPE and RMSE values, respectively. 
Table 17

Test dataset $\left(Z_{\text {test }}\right)$ for 2011 .

\begin{tabular}{|c|c|c|c|c|c|c|c|c|c|c|}
\hline Test & $\mathrm{a}$ & $\mathrm{b}$ & c & $\mathrm{d}$ & e & $\mathrm{f}$ & $\mathrm{g}$ & $\mathrm{h}$ & $\mathrm{i}$ & $\mathrm{j}$ \\
\hline 63210 & 62968.8 & 63220.3 & 62965.1 & 63201 & 62989.6 & 63574.9 & 62960.3 & 63418.5 & 63777.3 & 63427.7 \\
\hline 64561 & 62879.4 & 62686.5 & 62871.2 & 62430.2 & 62947.4 & 63099 & 63314.2 & 63018.6 & 62949.9 & 62495 \\
\hline 63609 & 64242.4 & 62754 & 64326.7 & 63591.5 & 64247.5 & 63438.4 & 64000 & 64042.1 & 63697 & 63443.6 \\
\hline 62407 & 63448.5 & 62946.6 & 63572.1 & 63351.6 & 63079.2 & 63255.5 & 62789 & 63642.4 & 64630.3 & 63538.2 \\
\hline 61492 & 62182.5 & 63391.2 & 62616.6 & 62117 & 62252.4 & 62962.3 & 62934.6 & 62161 & 62596.5 & 62527.2 \\
\hline 63046 & 61209.2 & 62083.6 & 61411.3 & 61396.2 & 61180.1 & 61326.9 & 62461.2 & 61357.6 & 63358.2 & 61181.7 \\
\hline MAPE & 0.0148 & 0.0140 & 0.0156 & 0.0134 & 0.0142 & 0.0138 & 0.0128 & 0.0132 & 0.0145 & 0.0159 \\
\hline
\end{tabular}

Table 18

Forecasting results and RMSE/MAPE values of existing methods and $M F F_{\text {best }}$.

\begin{tabular}{llllllll}
\hline Test & ARIMA & ES & MLP-ANN & FF & FTS-N & R-T1FF & MFF $_{\text {best }}$ \\
\hline 63210 & 63299 & 63298.61 & 63615.31 & 63500.67 & 63120.65 & 62597.67 & 63306.5 \\
64561 & 63210 & 63209.89 & 63782.23 & 63121.86 & 62925.94 & 62356.36 & 62872.82 \\
63609 & 64561 & 64561.3 & 64968.54 & 64690.38 & 63661.87 & 63079.91 & 63366.36 \\
63755 & 63609 & 63609.38 & 63569.83 & 63565.97 & 63571.09 & 63262.51 & 63161.82 \\
62407 & 63755 & 63755.34 & 64074.42 & 64245.59 & 63339.12 & 62956.39 & 63319.68 \\
61492 & 62407 & 62407.52 & 62966.45 & 62360.53 & 62617.5 & 62324.37 \\
63046 & 61492 & 61491.78 & 62465.25 & 61782.2 & 61648.56 & 61212.24 & 62680.64 \\
RMSE & 1057.62 & 1057.84 & 1065.36 & 1139.72 & 986.08 & 1202.09 & 935.82 \\
MAPE & 0.0144 & 0.0144 & 0.0147 & 0.0158 & 0.0123 & 0.0159 \\
\hline
\end{tabular}

Table 19

Training dataset $\left(Z_{\text {train }}\right)$ for 2012 .

\begin{tabular}{|c|c|c|c|c|c|c|c|c|c|c|}
\hline Test & $\mathrm{a}$ & $\mathrm{b}$ & c & $\mathrm{d}$ & e & f & $\mathrm{g}$ & $\mathrm{h}$ & $\mathrm{i}$ & $\mathrm{j}$ \\
\hline 58873 & 58571.8 & 58575.5 & 58375.1 & 58201.8 & 58338.4 & 58154.4 & 58719.7 & 58705.3 & 58547.4 & 58250.8 \\
\hline 57854 & 58645.8 & 58653.2 & 58757.9 & 58196.4 & 58406.5 & 58258.9 & 58774.4 & 58778.3 & 58565.7 & 57440.3 \\
\hline 58101 & 57235.6 & 57224.9 & 57299.3 & 57387.5 & 57354 & 57193.3 & 57377.1 & 57356.7 & 57262.9 & 58171 \\
\hline 57331 & 57899.7 & 57832.4 & 57846.7 & 57535.7 & 57614.9 & 57395.7 & 58087.9 & 58001.9 & 57751 & 57535.4 \\
\hline 56936 & 57137.4 & 57057.3 & 57176.5 & 57193.6 & 57223.2 & 57090.3 & 57330.1 & 57233.4 & 57137.4 & 56661.6 \\
\hline
\end{tabular}

Table 20

Test dataset $\left(Z_{\text {test }}\right)$ for 2012 .

\begin{tabular}{|c|c|c|c|c|c|c|c|c|c|c|}
\hline Test & $\mathrm{a}$ & $\mathrm{b}$ & c & $\mathrm{d}$ & e & $\mathrm{f}$ & $\mathrm{g}$ & $\mathrm{h}$ & $\mathrm{i}$ & $\mathrm{j}$ \\
\hline 55734 & 56852.8 & 56871.7 & 56786.3 & 56522.6 & 56599.4 & 56398.3 & 56968.1 & 56983.9 & 56741.9 & 56511 \\
\hline 54917 & 55519.1 & 55522.1 & 55527.7 & 55857.5 & 55734.9 & 55671 & 55646.4 & 55641 & 55573 & 55683.4 \\
\hline 54810 & 54702.5 & 54712.4 & 54644.7 & 55234 & 54955.1 & 54859.1 & 54820.8 & 54825.9 & 54768.6 & 55166.9 \\
\hline 54844 & 54612.6 & 54571.5 & 54646.7 & 54889.1 & 54653 & 54479.8 & 54783.6 & 54718.1 & 54593.9 & 54454.5 \\
\hline 55450 & 54682.3 & 54529.8 & 54757.1 & 54746.7 & 54591.6 & 54428.9 & 54981.8 & 54749.7 & 54594.5 & 54635.1 \\
\hline 55125 & 55288.7 & 55124.1 & 55369.1 & 55029.4 & 54950.9 & 54803 & 55613.4 & 55353.9 & 55091.7 & 55172 \\
\hline 55099 & 54979.2 & 54774.2 & 55121.9 & 55046.8 & 55003.1 & 54914.4 & 55365 & 55028.2 & 54936.7 & 54867.2 \\
\hline MAPE & 0.0080 & 0.0087 & 0.0077 & 0.0079 & 0.0081 & 0.0072 & 0.0084 & 0.0080 & 0.0078 & 0.0074 \\
\hline
\end{tabular}

Table 21

Forecasting results and RMSE/MAPE values of existing methods and $M F F_{\text {best }}$.

\begin{tabular}{|c|c|c|c|c|c|c|c|}
\hline Test & ARIMA & ES & MLP-ANN & $\mathrm{FF}$ & FTS-N & R-T1FF & $M F F_{\text {best }}$ \\
\hline 55734 & 57079 & 57079.46 & 57421.46 & 57781.04 & 56717.86 & 56461.83 & 56472.86 \\
\hline 54917 & 55734 & 55734.36 & 56014.45 & 56433.76 & 56055.82 & 55329.15 & 55338.75 \\
\hline 54810 & 54917 & 54916.62 & 55218.22 & 55638.09 & 55140.42 & 54546.99 & 54555.71 \\
\hline 55450 & 54844 & 54843.93 & 55185.19 & 55733.9 & 54811.12 & 54731.61 & 54726.21 \\
\hline 55125 & 55450 & 55449.5 & 55823.22 & 56244.47 & 55193.66 & 55193.92 & 55189.89 \\
\hline 55099 & 55125 & 55125.29 & 55452.13 & 55899.09 & 55181.59 & 55460.04 & 55439.44 \\
\hline MAPE & 0.0084 & 0.0084 & 0.01243 & 0.01945 & 0.0084 & 0.00739 & $0.00737^{*}$ \\
\hline
\end{tabular}

\subsection{Dow Jones datasets}

There are 4 datasets for Dow Jones index from 2010 to 2013. To obtain 10 models from R-T1FF, the number of clusters was varied from 2 to 5 , the AR lag was varied from 1 to 5 , and the MA lag was varied from 1 to 2 . The last 10 observations each Dow Jones datasets are selected as the test dataset $\left(Y_{\text {test }}\right)$. The input matrix $(Z)$ of MFFs are obtained from the 10 point forecasts of 10 models. The first 6 observations of $Z$ is selected as the training matrix $\left(Z_{\text {train }}\right)$ in order to determine the weights of the models in functions and $M F F_{\text {best }}$. The last 4 observations are left for test dataset $\left(Z_{\text {test }}\right)$ to evaluate the performance of the proposed method with the existing methods; ANFISgrid, ANFISsub, MANFIS [32], T1FF [5]. 
Table 22

Training dataset $\left(Z_{\text {train }}\right)$ for 2010 .

\begin{tabular}{|c|c|c|c|c|c|c|c|c|c|c|}
\hline Test & $\mathrm{a}$ & $\mathrm{b}$ & c & $\mathrm{d}$ & e & $\mathrm{f}$ & $\mathrm{g}$ & $\mathrm{h}$ & $\mathrm{i}$ & $\mathrm{j}$ \\
\hline 11491.91 & 11509.24 & 11496.92 & 11447.94 & 11508.6 & 11504.61 & 11501.48 & 11506.74 & 11542.65 & 11524.84 & 11461.46 \\
\hline 11478.13 & 11496.21 & 11513 & 1149 & 1149 & 114 & 115 & 1151 & .08 & & \\
\hline 11533.16 & 11486.68 & 11499.99 & 11420.47 & 11485.82 & 1148 & 11487.25 & 114 & 4.27 & 1149 & 11469.43 \\
\hline 11573.49 & 11584.82 & 11559.2 & 11538.18 & 11560.06 & & 11559.64 & & & 11571.88 & 11532.97 \\
\hline 11555.03 & 11621.4 & 11580.19 & 11567.66 & 11564.82 & 11560.63 & 11574.13 & 11581.22 & 11579.32 & 11573.67 & 11545.42 \\
\hline
\end{tabular}

Table 23

Test dataset $\left(Z_{\text {test }}\right)$ for 2010 .

\begin{tabular}{|c|c|c|c|c|c|c|c|c|c|c|}
\hline Test & a & b & c & $\mathrm{d}$ & e & $\mathrm{f}$ & g & $\mathrm{h}$ & i & $\mathrm{j}$ \\
\hline 11575.54 & 11587.86 & 11579.73 & 11529.32 & 11558.87 & 11556.17 & 11556.73 & 11565.74 & 11613.85 & 11569.1 & 11572.72 \\
\hline 11585.38 & 11633.49 & 11587.79 & 11559.15 & 11565.57 & 11558.74 & 11572.06 & 11578.47 & 11647.2 & 11570.81 & 11552.09 \\
\hline 11569.71 & 11661.2 & 11594.12 & 11577.85 & 11568.09 & 11566.77 & 11584.86 & 11591.76 & 11616.61 & 11577.46 & 11583.81 \\
\hline 11577.51 & 11600.34 & 11595.19 & 11539.11 & 11583.51 & 11577.85 & 11569.66 & 11578.07 & 11635.35 & 11591.52 & 11585.33 \\
\hline RMSE & 53.287 & 15.263 & 33.035 & 13.313 & 16.535 & 14.339 & 12.553 & 52.044 & 11.293 & 18.548 \\
\hline MAPE & 0.00377 & 0.00105 & 0.00257 & 0.00095 & 0.00106 & 0.00119 & 0.00085 & 0.00442 & 0.00092 & 0.00125 \\
\hline
\end{tabular}

Table 24

Weights of the models in functions and RMSE values of the functions for $Z_{\text {train }}$.

\begin{tabular}{lllll}
\hline Model & $M F F_{1}$ & $M F F_{2}$ & $M F F_{3}$ & $M F F_{4}$ \\
\hline a & 0 & 0 & 0 & 0.987 \\
b & 0.165 & 0 & 0 & 0 \\
c & 0 & 0 & 0.476 & 0 \\
d & 0.167 & 0 & 0 & 0 \\
e & 0.165 & 0 & 0 & 0 \\
$\mathrm{f}$ & 0.167 & 0 & 0 & 0 \\
g & 0.167 & 0.001 & 0.001 & 0.002 \\
h & 0 & 0.992 & 0 & 0 \\
i & 0.165 & 0.004 & 0.001 & 0.009 \\
j & 0.005 & 0.003 & 0.522 & 0.002 \\
\hline RMSE & $24.93^{*}$ & 35.00 & 46.44 & 34.81 \\
MAPE & $0.0020^{*}$ & 0.0028 & 0.0034 & 0.0024 \\
\hline
\end{tabular}

Table 25

Forecasts of MFFs for 2010 and RMSE and MAPE values of the functions.

\begin{tabular}{cllll}
\hline Model & $M F F_{1}{ }^{*}$ & $M F F_{2}$ & $M F F_{3}$ & $M F F_{4}$ \\
\hline 7 & 11564.4 & 11613.49 & 11552.04 & 11587.61 \\
8 & 11572.12 & 11646.52 & 11555.49 & 11632.63 \\
9 & 11580.52 & 11616.32 & 11580.97 & 11660.12 \\
10 & 11582.6 & 11634.96 & 11563.32 & 11600.18 \\
\hline RMSE & $10.52^{*}$ & 51.60 & 21.06 & 52.60 \\
MAPE & $0.0009^{*}$ & 0.0044 & 0.0017 & 0.0037 \\
\hline
\end{tabular}

The number of clusters is varied from 2 to 5 and the fuzziness value is varied from 1.3 to 3 with the increase rate of 0.1 for MFFs. Although the detailed results are given for the dataset of 2010, only the input matrices and formal comparison results are given for 2011-2013.

\subsubsection{0}

For Dow Jones dataset in 2010, 10 forecasts of 10 models are used as the input matrix, $Z$. The first 6 observations of $Z$ is used as training matrix $\left(Z_{\text {train }}\right)$ and the last 4 observations are used as the test dataset $\left(Z_{\text {test }}\right)$ and they are given in Tables 22 and 23, respectively.

The best MFFs are obtained when $m=1.4$ and $c=4$. The weight matrix that are calculated by FCM for $Z_{\text {train }}$ is given in Table 24. The best function is determined from MAPE values of the MFFs for $Z_{\text {train }}$. In this case, $M F F_{1}$ is selected as $M F F_{\text {best }}$. Thus, the forecasts of the proposed methods is obtained by using $M F F_{b e s t}$. $M F F_{\text {best }}$ is given in Eq. (30) and the corresponding forecasting results and the results of other functions are given in Table 25.

$M F_{\text {best }}=M F_{1}=0.165 * a+\cdots+0.165 * i+0.005 * j$
Table 26

Forecasting results and RMSE/MAPE values of existing methods and $M F F_{\text {best }}$.

\begin{tabular}{llllll}
\hline Test & ANFISgrid & ANFISsub & MANFIS & T1FF & MFF $_{\text {best }}$ \\
\hline 11575.54 & 11571.59 & 11587.34 & 11563.86 & 11562.97 & 11564.4 \\
11585.38 & 11601.37 & 11606.63 & 11584.40 & 11580.95 & 11572.12 \\
11569.71 & 11591.92 & 11617.79 & 11594.25 & 11589.56 & 11580.52 \\
11577.51 & 11546.67 & 11606.31 & 11578.56 & 11575.85 & 11582.6 \\
\hline RMSE & 20.71 & 30.54 & 13.61 & 11.98 & 10.52 \\
MAPE & 0.0016 & 0.0024 & 0.0008 & 0.0008 & 0.0008 \\
\hline
\end{tabular}

4 existing forecasting methods are compared with the proposed method in Table 26. It is obvious that the $M F F_{\text {best }}$ of the proposed methods outperforms other methods in terms of both RMSE and MAPE values.

\subsubsection{1}

There are 251 observations in Dow Jones dataset for 2011 and the last 10 observations are taken as the test dataset $\left(Y_{\text {test }}\right)$. Using $Y_{\text {train }}, 10$ models are obtained from R-T1FF with different parameter specifications. In order to obtain the input matrix $(Z)$ of MFFs, 10 forecast are calculated for each model that are obtained from R-T1FFs. The first 6 forecasts of the 10 models are used as the training $\left(Z_{\text {train }}\right)$ and the rest of the forecasts are used as the test $\left(Z_{\text {test }}\right)$ datasets. Training and test sets are given in Tables 27-28, respectively.

The best MFFs are obtained when $m=1.4$ and $c=5$. The weights of the models in functions are obtained by using the degree of membership values of the models in clusters for $Z_{\text {train }}$. The best function $\left(M F F_{b e s t}\right)$ is determined as the fifth meta fuzzy function and given in Eq. (31).

$$
\begin{aligned}
M F F_{\text {best }}=M F F_{5}= & 0.85 * b+0.143 * g \\
& +0.003 h+0.01 * i+0.03 * j
\end{aligned}
$$

We are able to say that the performance of the proposed method is better than all existing forecasting methods in terms of RMSE and MAPE values in Table 29.

\subsubsection{2}

The 10 forecasts of the 10 models are used as the input matrix $(Z)$ of MFFs. The input matrix is divided into two training $\left(Z_{\text {train }}\right)$ and test $\left(Z_{\text {test }}\right)$ sets. Tables $30-31$ represents the training and test sets, respectively.

Training dataset is clustered by using FCM and the weights of the models that are calculated from the degrees of memberships are obtained for each function. The best set of meta fuzzy functions 
Table 27

Training dataset $\left(Z_{\text {train }}\right)$ for 2011

\begin{tabular}{|c|c|c|c|c|c|c|c|c|c|c|}
\hline Test & a & b & c & d & e & f & $\mathrm{g}$ & h & $\mathrm{i}$ & $\mathrm{j}$ \\
\hline 11866.39 & 11857 & 11855.71 & 11897.39 & 11850.31 & 11878.25 & 11882.41 & 11926.76 & 11972.49 & 11833.98 & 11875.74 \\
\hline 11766.26 & 11854.57 & 11885.18 & 11892.79 & 11838.86 & 11860.4 & 11866.2 & 11907.15 & 11934.58 & 55.61 & 11860.47 \\
\hline 12103.58 & 11749.06 & 11949.04 & 11789.68 & 11718.03 & 11748.25 & 11758.53 & 11840.78 & 11854.75 & 11760.46 & 11752.51 \\
\hline 12169.65 & 12098.85 & 12423.39 & 12133.78 & 12094.52 & 12086.54 & 12103.93 & 12232.23 & 12183.75 & 12082.13 & 12088.14 \\
\hline 12294 & 12165.39 & 12345.28 & 12196.03 & 12190.67 & 12193.46 & 12192.07 & 12211.43 & 12236.02 & 12125 & 12190.63 \\
\hline
\end{tabular}

Table 28

Test dataset $\left(Z_{\text {test }}\right)$ for 2011 .

\begin{tabular}{|c|c|c|c|c|c|c|c|c|c|c|}
\hline Test & $\mathrm{a}$ & b & c & d & $\mathrm{e}$ & $\mathrm{f}$ & $\mathrm{g}$ & h & i & $\mathrm{j}$ \\
\hline 12291.35 & 12283.01 & 12349.38 & 12320.18 & 12265.27 & 12302.62 & 12302.62 & 12292.61 & 12375.88 & 12276.75 & 12296.55 \\
\hline 12151.41 & 12266.55 & 12295.66 & 12316.39 & 12218.12 & 12280.77 & 12286.05 & 12315.51 & 12387.26 & 12300.29 & 12282.49 \\
\hline 12287.04 & 12135.2 & 12271.84 & 12176.54 & 12118.61 & 12132.35 & 12143.89 & 12185.22 & 12235.38 & 12141.79 & 12141.97 \\
\hline 12217.56 & 12258.35 & 12254.01 & 12311.6 & 12194.92 & 12270.37 & 12260.35 & 12250.39 & 12387.7 & 12302.51 & 12259.55 \\
\hline RMSE & 97.53 & 80.21 & 110.80 & 92.21 & 104.38 & 100.72 & 97.95 & 153.61 & 112.57 & 100.02 \\
\hline MAPE & 0.0065 & 0.0052 & 0.0082 & 0.0058 & 0.0071 & 0.0068 & 0.0061 & 0.0111 & 0.0081 & 0.0066 \\
\hline
\end{tabular}

Table 29

Forecasting results and RMSE/MAPE values of existing methods and $M F F_{\text {best }}$.

\begin{tabular}{llllll}
\hline Test & ANFISgrid & ANFISsub & MANFIS & T1FF & MFF best \\
\hline 12291.35 & 12268.61 & 12329.13 & 12285.16 & 12271.36 & 12341.09 \\
12151.41 & 12285.36 & 12296.95 & 12319.05 & 12285.72 & 12298.72 \\
12287.04 & 12115.69 & 12149.06 & 12218.30 & 12150.31 & 12258.83 \\
12217.56 & 12259.14 & 12290.89 & 12274.94 & 12261.81 & 12253.92 \\
\hline RMSE & 111.30 & 108.43 & 95.08 & 98.86 & 81.07 \\
MAPE & 0.0076 & 0.0081 & 0.0061 & 0.0069 & 0.0054 \\
\hline
\end{tabular}

are obtained when $m=3$ and $c=5$. The best meta fuzzy function is detected as the third function and it is given in Eq. (32).

$$
\begin{aligned}
M_{\text {best }}=\mathrm{MFF}_{3}= & 0.85 * b+0.143 * g \\
& +0.003 h+0.01 * i+0.03 * j
\end{aligned}
$$

Table 32 is given to compare the results of the existing methods and the proposed method. The table shows that the proposed method outperforms the other methods in terms of both RMSE and MAPE values.

\subsubsection{3}

For 2013, the training and test datasets for MFFs are given in Tables 33-34, respectively. The best set of MFFs are obtained when $m=2$ and $c=4$. The best function is determined as the fourth function for $Z_{\text {train }}$. Thus the $M F F_{\text {best }}$ is the fourth function of the MFFs and given in Eq. (33).

$M F F_{\text {best }}=M \mathrm{MF}_{4}=0.39 * c+0.304 * g+0.278 * h$
Table 32

Forecasting results and RMSE/MAPE values of existing methods and $M F F_{\text {best }}$.

\begin{tabular}{llllll}
\hline Test & ANFISgrid & ANFISsub & MANFIS & T1FF & MFF $_{\text {best }}$ \\
\hline 13114.59 & 13171.73 & 13119.31 & 13152.26 & 13111.70 & 13074.81 \\
13096.31 & 13183.93 & 13091.44 & 13123.75 & 13091.97 & 13090.32 \\
12938.11 & 12982.05 & 13070.81 & 13104.55 & 13082.15 & 13029.86 \\
13104.14 & 12947.44 & 12929.84 & 12966.91 & 12956.00 & 12949.25 \\
\hline RMSE & 96.73 & 109.59 & 110.35 & 103.34 & 92.23 \\
MAPE & 0.0066 & 0.0061 & 0.0071 & 0.0057 & 0.0056 \\
\hline
\end{tabular}

$$
+0.015 * i+0.013 * j
$$

Table 35 is given to compare the results of the existing methods and the proposed method. The table shows that the proposed method has better forecasting performances in terms of both RMSE and MAPE values.

\section{Conclusions}

A naive approach based on FCM is introduced to aggregate the methods in the paper. MFFs are able to cluster the methods in functions. For example, while the methods that perform better for a dataset is collected in a function, the methods that perform worse is collected in a different function with certain degrees of memberships values. The advantages of the MFFs are listed below.

- MFFs is the first introduced approach that aims to aggregate methods into functions. MFFs are introduced with an assumption that each method has its own understanding of a

\begin{tabular}{|c|c|c|c|c|c|c|c|c|c|c|}
\hline Test & $a$ & b & c & d & e & $\mathrm{f}$ & $\mathrm{g}$ & $\mathrm{h}$ & $\mathrm{i}$ & $\mathrm{j}$ \\
\hline 13235.39 & 13056.98 & 13176.11 & 13038.39 & 13073.12 & 13113.15 & 13209.58 & 13235.87 & 13148.85 & 13073.29 & 13042.41 \\
\hline 13350.96 & 13224.84 & 13246.35 & 13233.24 & 13228.72 & 13229.49 & 13345.16 & 13284.5 & 13224.6 & 13161.99 & 13147.94 \\
\hline 13251.97 & 13278.11 & 13383.79 & 13252.59 & 13290.75 & 13346.29 & 13438.05 & 13308.66 & 13340.09 & 13276.86 & 13330.3 \\
\hline 13311.72 & 13246.44 & 13274.32 & 13257.43 & 13255.6 & 13223.93 & 13308.26 & 13235.23 & 13283.47 & 13250.49 & 13274.17 \\
\hline 13190.84 & 13239.1 & 13338.54 & 13209.6 & 13247.12 & 13301.04 & 13367.06 & 13427.85 & 13304.08 & 13219.84 & 13175.33 \\
\hline 13139.08 & 13168.5 & 13207.68 & 13190.34 & 13178.6 & 13157.27 & 13262.86 & 13198.67 & 13206.96 & 13191.14 & 13218.59 \\
\hline
\end{tabular}

Table 30

Training dataset $\left(Z_{\text {train }}\right)$ for 2012 .

\begin{tabular}{|c|c|c|c|c|c|c|c|c|c|c|}
\hline Test & $\mathrm{a}$ & b & c & d & e & f & g & h & $\mathrm{i}$ & $\mathrm{j}$ \\
\hline 13114.59 & 13071.78 & 13163.95 & 13035.07 & 13077.51 & 13114.57 & 13218.85 & 13262.62 & 13153.67 & 13074.91 & 13152.67 \\
\hline 13096.31 & 13086.45 & 13131.89 & 13115.85 & 13098.23 & 13093.7 & 13189.57 & 13185.82 & 13115.04 & 13077.62 & 12982.81 \\
\hline 12938.11 & 13039.59 & 13123.11 & 12994.85 & 13044.86 & 13076.02 & 13163.45 & 13142.86 & 13103.29 & 13043.67 & 13081.43 \\
\hline 13104.14 & 12899.95 & 12952.87 & 12937.57 & 12911.22 & 12897.47 & 13005.45 & 13001.49 & 12965.96 & 12922.84 & 13038.9 \\
\hline RMSE & 116.11 & 123.30 & 97.04 & 111.79 & 124.24 & 141.50 & 143.51 & 109.84 & 107.16 & 98.91 \\
\hline MAPE & 0.0069 & 0.0081 & 0.0062 & 0.0065 & 0.0067 & 0.0100 & 0.0104 & 0.0069 & 0.0066 & 0.0069 \\
\hline
\end{tabular}

Table 31

Test dataset $\left(Z_{\text {test }}\right)$ for 2012 . 
Table 33

Training dataset $\left(Z_{\text {train }}\right)$ for 2013

\begin{tabular}{|c|c|c|c|c|c|c|c|c|c|c|}
\hline Test & $\mathrm{a}$ & $\mathrm{b}$ & c & $\mathrm{d}$ & $\mathrm{e}$ & $\mathrm{f}$ & $\mathrm{g}$ & $\mathrm{h}$ & $\mathrm{i}$ & $\mathrm{j}$ \\
\hline 15875.26 & 15924.92 & 15903.44 & 15919.36 & 15935.81 & 15856.47 & 15989.79 & 15908.92 & 15982.18 & 15832.66 & 15858.63 \\
\hline 16167.97 & 15920.97 & 15896.86 & 15920.98 & 15898.55 & 15840.76 & 15985.11 & 15949.97 & 15926.9 & 15881.37 & 15887.72 \\
\hline 16179.08 & 16200.91 & 16184.55 & 16218.42 & 16206.11 & 16146.1 & 16280.69 & 16140.39 & 16242.17 & 16043.13 & 16095.08 \\
\hline 16221.14 & 16210.18 & 16194.1 & 16230.46 & 16210.97 & 16163.67 & 16291.27 & 16241.09 & 16243.89 & 16170.52 & 16178.51 \\
\hline 16294.61 & 16257.8 & 16237.63 & 16272.24 & 16258.9 & 16200.72 & 16332.8 & 16277.16 & 16292 & 16207.91 & 16220.33 \\
\hline 16357.55 & 16331.07 & 16311.87 & 16346.56 & 16326.83 & 16269.35 & 16407.64 & 16344.89 & 16361.56 & 16266.5 & 16286.06 \\
\hline
\end{tabular}

Table 34

Test dataset $\left(Z_{\text {test }}\right)$ for 2013 .

\begin{tabular}{|c|c|c|c|c|c|c|c|c|c|c|}
\hline Test & $\mathrm{a}$ & b & c & $\mathrm{d}$ & $\mathrm{e}$ & f & $\mathrm{g}$ & h & $\mathrm{i}$ & $\mathrm{j}$ \\
\hline 16479.88 & 16390.09 & 16374.52 & 16410.98 & 16389.69 & 16333.34 & 16472.29 & 16415.27 & 16424.57 & 16332.66 & 16352.38 \\
\hline 16478.41 & 16511.81 & 16496.75 & 16534.34 & 16511.73 & 16458.25 & 16595.82 & 16518.32 & 16547.13 & 16427.8 & 16457.44 \\
\hline 16504.29 & 16511.01 & 16495.34 & 16533.47 & 16509.64 & 16456.77 & 16594.71 & 16562.95 & 16545 & 16481.17 & 16492.45 \\
\hline 16576.66 & 16536.43 & 16521.29 & 16560 & 16534.83 & 16481.1 & 16621.23 & 16581.48 & 16570.57 & 16498.68 & 16514.53 \\
\hline RMSE & 52.06 & 60.38 & 47.45 & 52.49 & 91.20 & 77.47 & 48.04 & 48.67 & 87.82 & 71.93 \\
\hline MAPE & 0.0026 & 0.0028 & 0.0026 & 0.0026 & 0.0047 & 0.0039 & 0.0025 & 0.0026 & 0.0045 & 0.0034 \\
\hline
\end{tabular}

Table 35

Forecasting results and RMSE/MAPE values of existing methods and $M F F_{\text {best }}$.

\begin{tabular}{llllll}
\hline Test & ANFISgrid & ANFISsub & MANFIS & T1FF & MFF $_{\text {best }}$ \\
\hline 16479.88 & 16236.68 & 16281.99 & 16377.62 & 16390.06 & 16414.16 \\
16478.41 & 16353.20 & 16365.03 & 16495.93 & 16507.48 & 16530.48 \\
16504.29 & 16214.60 & 16345.41 & 16503.17 & 16510.77 & 16544.35 \\
16576.66 & 16256.59 & 16357.09 & 16527.16 & 16536.35 & 16567.99 \\
\hline RMSE & 255.53 & 177.11 & 57.48 & 51.43 & 46.66 \\
MAPE & 0.0148 & 0.0104 & 0.0026 & 0.0025 & 0.0025 \\
\hline
\end{tabular}

dataset. For example, for a specific purpose like forecasting or predicting, there are many methods in literature mainly using the same arguments with different modifications. Therefore, our assumption is that each method has more or less knowledge for a given dataset.

- One does not need to have a deep understanding of fuzzy set theory to implement or understand the proposed method. The only need for implementing the proposed method is to understand the FCM algorithm.

- MFFs guarantees, at least, the best performance of the methods that are used. In another word, MFFs are also able to search for the best method among many. However, MFFs usually increase the performance of the related methods by aggregating them into functions in terms of some evaluation criteria.

Although the aim of the study is to aggregate the methods for a specific purpose, the models of a method with different parameter specifications are aggregated as an application in the paper. 9 real world datasets were chosen for the evaluation. Results showed that MFFs are able to increase the performance of R-T1FFs. For all nine datasets, the best performances for forecasting in terms of both RMSE and MAPE values were obtained from the MFFs approach. Besides, comparing the results of the proposed method with other alternative methods in literature, it can be said that MFFs based R-T1FFs outperforms most of them in terms of both RMSE and MAPE. Aggregating methods for forecasting and employing the possibilistic FCM are left for the future work. Besides, aggregation of regression methods and indices are another future works.

\section{Acknowledgment}

I would like to thank to the handling editor Prof. Dr. Oscar Castillo and the reviewers for their helpful comments and suggestions. And, also, I would like to thank to the people of Forecast Research Laboratory of Giresun University for their comments and suggestions.

\section{References}

[1] G.V. Glass, Primary, secondary, and meta-analysis of research, Educ. Researcher 5 (10) (1976) 3-8, http://dx.doi.org/10.3102/0013189X005010003.

[2] R. DerSimonian, N. Laird, Meta-analysis in clinical trials, Controlled Clin. Trials 7 (3) (1986) 177-188, http://dx.doi.org/10.1016/0197-2456(86)90046-2.

[3] J.C. Bezdek, R. Ehrlich, W. Full, FCM: The fuzzy c-means clustering algorithm, Comput. Geosci. 10 (2-3)(1984) 191-203, http://dx.doi.org/10.1007/s10898007-9149-X.

[4] N. Tak, A.A. Evren, M. Tez, E. Egrioglu, Recurrent type-1 fuzzy functions approach for time series forecasting, Appl. Intell. 48 (1) (2018) 68-77, http: //dx.doi.org/10.1007/s10489-017-0962-8.

[5] I.B. Turksen, Fuzzy functions with LSE, Appl. Soft Comput. 8 (3) (2008) 1178-1188, http://dx.doi.org/10.1016/j.asoc.2007.12.004.

[6] S. Beyhan, M. Alci, Fuzzy functions based ARX model and new fuzzy basis function models for nonlinear system identification, Appl. Soft Comput. 10 (2010) 439-444, http://dx.doi.org/10.1016/j.asoc.2009.08.015.

[7] C.H. Aladag, I.B. Turksen, A.Z. Dalar, E. Egrioglu, U. Yolcu, Application of type-1 fuzzy functions approach for time series forecasting, Turkish J. Fuzzy Syst. 5 (1) (2014) 1-9.

[8] R. Krishnapuram, J.M. Keller, A possibilistic approach to clustering, IEEE Trans Fuzzy Syst. 1 (2) (1993) 98-110, http://dx.doi.org/10.1109/91.227387.

[9] N.R. Pal, K. Pal, J.M. Keller, J.C. Bezdek, A possibilistic fuzzy c-means clustering algorithm, IEEE Trans. Fuzzy Syst. 13 (4) (2005) 517-530, http://dx.doi.org/ 10.1109/TFUZZ.2004.840099.

[10] E. Rubio, O. Castillo, F. Valdez, P. Melin, C.I. Gonzalez, G. Martinez, An extension of the fuzzy possibilistic clustering algorithm using type-2 fuzzy logic techniques, Adv. Fuzzy Syst. 2017 (2017) 1-23, http://dx.doi.org/10.1155/ 2017/7094046.

[11] Q. Song, B.S. Chissom, Forecasting enrollments with fuzzy time series - Part I, Fuzzy Sets and Systems 54 (1) (1993) 1-9, http://dx.doi.org/10.1016/01650114(93)90355-L.

[12] Q. Song, B.S. Chissom, Forecasting enrollments with fuzzy time series - Part II, Fuzzy Sets and Systems 62 (1) (1994) 1-8, http://dx.doi.org/10.1016/01650114(94)90067-1.

[13] S.M. Chen, Forecasting enrollments based on fuzzy time series, Fuzzy Sets and Systems 81 (3) (1996) 311-319, http://dx.doi.org/10.1016/0165-0114(95) 00220-0.

[14] D.W. Chen, J.P. Zhang, Time series prediction based on ensemble ANFIS in: Machine Learning and Cybernetics, Proceedings of 2005 International Conference, vol. 6, 2005, pp. 3552-3556, http://dx.doi.org/10.1109/ICMLC. 2005.1527557.

[15] C. Gupta, A. Jain, D.K. Tayal, O. Castillo, Forecasting low dimensional numerical data using an improved method based on automatic clustering, fuzzy relationships and differential evolution, Eng. Appl. Artif. Intell. 71 (2018) 175-189, http://dx.doi.org/10.1016/j.engappai.2018.02.015.

[16] C.H. Aladag, U. Yolcu, E. Egrioglu, A.Z. Dalar, A new time invariant fuzzy time series forecasting method based on particle swarm optimization, Appl. Soft Comput. 12 (10)(2012)3291-3299, http://dx.doi.org/10.1016/j.asoc.2012.05 002.

[17] S. Makridakis, Why combining works? Int. J. Forecast. 5 (4) (1989) 601-603, http://dx.doi.org/10.1016/0169-2070(89)90017-4.

[18] J.M. Bates, C.W. Granger, The combination of forecasts, J. Oper. Res. Soc. 20 (4) (1969) 451-468, http://dx.doi.org/10.1057/jors.1969.103.

[19] C.W. Granger, R. Ramanathan, Improved methods of combining forecasts, J. Forecast. 3 (2) (1984) 197-204, http://dx.doi.org/10.1002/for.3980030207. 
[20] D.W. Bunn, A Bayesian approach to the linear combination of forecasts, J. Oper. Res. Soc. 26 (2) (1975) 325-329, http://dx.doi.org/10.1057/jors.1975.67.

[21] P. Newbold, C.W. Granger, Experience with forecasting univariate time series and the combination of forecasts, J. R. Stat. Soc. Ser. A (1974) 131-165, http: //dx.doi.org/10.2307/2344546.

[22] H. Zou, Y. Yang, Combining time series models for forecasting, Int. J. Forecast 20 (1) (2004) 69-84, http://dx.doi.org/10.1016/S0169-2070(03)00004-9.

[23] C.H. Aladag, E. Egrioglu, U. Yolcu, Forecast combination by using artificial neural networks, Neural Process. Lett. 32 (3) (2010) 269-276, http://dx.doi. org/10.1007/s11063-010-9156-7.

[24] N.R. Pal, J.C. Bezdek, On cluster validity for the fuzzy c-means model, IEEE Trans. Fuzzy Syst. 3 (3) (1995) 370-379, http://dx.doi.org/10.1109/91.413225.

[25] I. Ozkan, I.B. Turksen, Upper and lower values for the level of fuzziness in FCM Inform. Sci. 177 (23) (2007) 5143-5152, http://dx.doi.org/10.1016/j.ins.2007. 06.028.

[26] K.P. Chan, Y.S. Cheung, Clustering of clusters, Pattern Recognit. 25 (2) (1992) 211-217, http://dx.doi.org/10.1016/0031-3203(92)90102-0.

[27] J.C. Bezdek, A physical interpretation of fuzzy ISODATA, Read. Fuzzy Sets Intell. Syst. (1993) 615-616, http://dx.doi.org/10.1016/B978-1-4832-14504.50065- 1 .
[28] G.J. Janacek, Practical Time Series, Oxford University Press Inc., New York, 2001.

[29] BIST100, Istanbul stock exchange index dataset, 2015. http://www.borsaista nbul.com/veriler/gecmise-donuk-veri-satisi. (Accessed 5 November 2015).

[30] G.E. Box, G.M. Jenkins, G.C. Reinsel, G.M. Ljung, Time Series Analysis: Forecasting and Control, John Wiley and Sons, 2015.

[31] J.S. Jang, ANFIS: Adaptive-network-based fuzzy inference systems, IEEE Trans. Syst. Man Cybern. 23 (3) (1993) 665-685, http://dx.doi.org/10.1109/21. 256541.

[32] E. Egrioglu, C.H. Aladag, U. Yolcu, E. Bas, A new adaptive network based fuzzy inference system for time series forecasting, Aloy J. Soft Comput. Appl. 2 (2014) 25-32.

[33] B. Sarica, E. Egrioglu, B. Asikgil, A new hybrid method for time series forecasting: Aranfis, Neural Comput. Appl. 29 (3) (2018) 749-760, http://dx.doi.org/ 10.1007/s00521-016-2475-5.

[34] E.S. Gardner, Exponential smoothing: The state of the art, J Forecast. 4 (1) (1985) 1-28, http://dx.doi.org/10.1002/for.3980040103.

[35] E. Bas, E. Egrioglu, C.H. Aladag, U. Yolcu, Fuzzy-time-series network used to forecast linear and nonlinear time series, Appl. Intell. 43 (2) (2015) 343-355, http://dx.doi.org/10.1007/s 10489-015-0647-0. 